\title{
THE PHILOSOPHY AND DRIVING FORCES IN THE DEVELOPMENT OF MEASUREMENT SCIENCE - SOME VIEWS UPON THE PROBLEMS (IDAACS'2001)
}

\author{
Ivan Kalchev
}

Technical University of Sofia, Faculty of Automatics BG-1797 Sofia, BULGARIA

E-mail: idk@tu-sofia.acad.bg

\begin{abstract}
A necessary background for clear outlined existing of each science in the so fast changing world is its permanent development in theoretical and applied aspect for solving today's problems and visions in the future. In this respect for the modern sciences, more important role play theories, that not only generalize and explain the known facts and phenomena, but serve for predicting and achieving new levels of knowledge.

In this paper the methods of teaching in the discipline Distributed Measurement Systems and in particular, the laboratory exercises carrying out by the students of Faculty of Automation and English Language Department of Engineering in Technical University of Sofia are presented.
\end{abstract}

Keywords: measurement science, driving forces, problems of teaching,

\section{MOTIVATIONS FOR THE DEVELOPMENT OF MEASUREMENT SCIENCE}

Signal and Information Theory is one of the basic aspects of Measurement science. Its role consists in the fact that the modern technology, underlying measurement needs new methods both for modeling of measurement systems at design stage, and for carrying out the so-called model-based measurement.

Model-based measurement consists of interrogating the system under observation by exciting it and estimating the quantities under observation as parameters of a model of the system.

This problem of system identification is a specifically measurement problem. The control of measurement is an essential condition to secure the quality of products, too.

There is three levels evolution of quality management:

- End Control System;

- Process Control Quality System;

- Total Quality Management.

The End Control System is the lowest level of quality management. Here, all products are object of quality inspection.
These are activities such as measuring, examining, testing, gauging one or more characteristics of a product or service and comparing these with specified requirements to determine conformity. The characteristics of this stage are: identification of sources of non-conformance, corrective actions, sorting, grading, re-blending, and salvage.

Basically the quality systems can be broken down into two types:

- Detection-based quality systems (fig. 1)

- Prevention-based quality systems (fig. 2)

The detection-based quality system corresponds to the first level of quality management evolution. In the prevention-based quality systems there are mechanis ms allowing the necessary corrective actions to be taken, not only at the end of the produc-

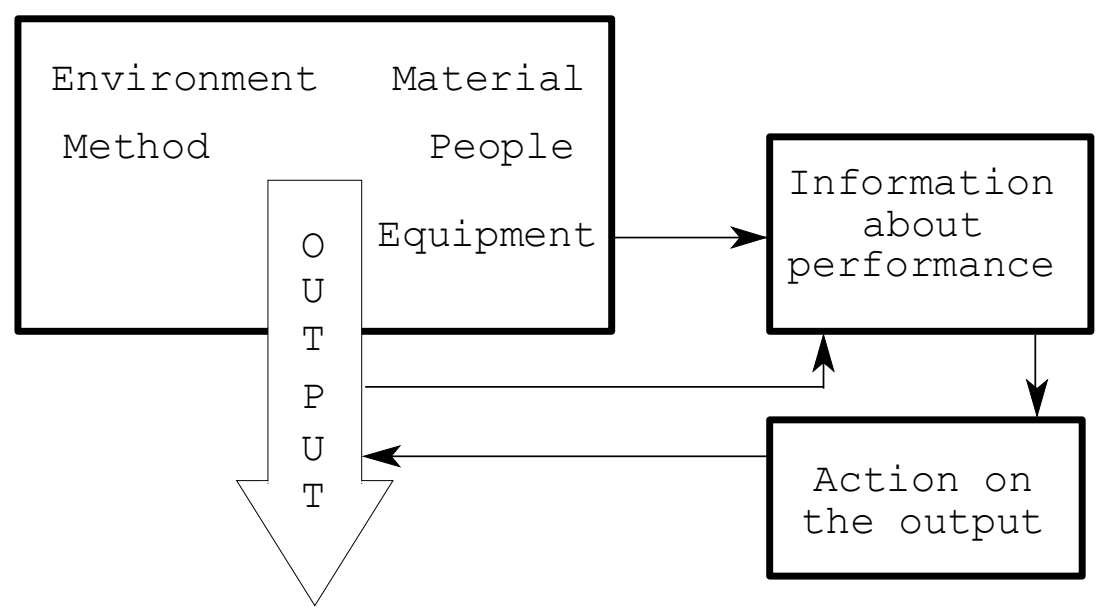

Fig. 1. Detection-based quality system 


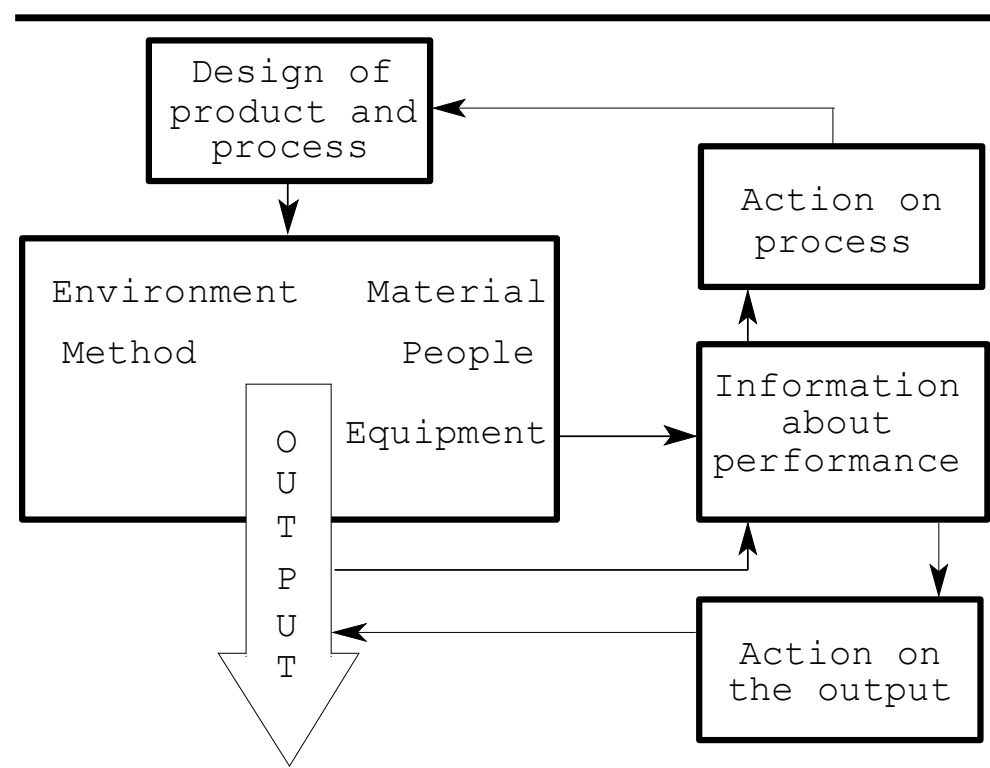

Fig. 2. Prevention-based quality system combined: quality control and quality as surance.

The most important method for this aim is the so-called statistical method for quality control. It becomes possible not only to check the product, but also to search for the causes of non-conformance and if there is a variation in the process parameters, it has to be corrected. Then the end result will be conformable to the quality standards (for example ISO 9001, ISO 9002, and ISO 9003).

The emphasis in the third level Total Quality Management (TQM) is placed on preventive mechanisms.

In the context of motivations for the development it is necessary to focus tion process, but before it has started, even at the design stage.

In the field of quality control it should be mentioned Pareto diagrams. Pareto diagram is a technique supporting the quality improvement process for ordering causes or problems from the most to the least significant. In this way the most significant aspects are identified and the efforts can be concentrated on those, thus getting the maximum benefit with the least effort. It is a special case of bar chart method and is used in conjunction with brainstorming, and cumulative line charts. The Pareto diagram is based on the Pareto principle, which states that a few causes account for most of the effect. It is usually applied for quality improvement: solving a few key quality problems can lead to major improvements.

It can be used the next algorithm for constructing Pareto diagrams:

Step 1. Decide the problem, which is to be analyzed.

Step 2. Decide the period over which the data are to be collected.

Step 3. Identify the main causes or categories of the problem.

Step 4. Collect the data using for example a check sheet.

Step 5. Tabulate the frequency of each category and list in descending order of frequency.

Step 6. Arrange the data as in a bar chart.

Step 7. Construct the Pareto diagram with the columns arranged in order of descending frequency.

Step 8. Determine commutative totals and percentages and construct the commutative percent curve.

Two very important aspects of quality control at the Process Control Quality System level are the attention on progress in the Theory of errors and uncertainty inmeasurement. They are specially important to measurement processes.

Many authors consider the need to develop the technology of measurement and instrumentation into an university discipline which can be effectively taught as the most powerful driving force behind the development of measurement science.

\section{THE PHILOSOPHY OF THE LECTURES ON DIST RIBUTED MEASUREMENT SYSTEMS}

The main purpose in creating of the lectures and exercises on distributed instrumentation was to model the basic principles of microprocessor-based measurement systems: constructing of primary sensors, signal conditioning elements, Analog to Digital Conversion (ADC), controllers for 16 and 32bit microprocessors and estimation of the final result for the measured value based on inverse transfer function. The problems of processing of repeatedly-trial measurement schemes are separately considered.

An essential part of the lectures is devoted to the estimation of measurement information. A crucial characteristic of the data obtained in the measurement process is their us efulness.

If we have a set of observatio
$w=(w 1, \ldots, w N) T$,

where:

$T$ - denotes transposition, since all values are considered as real,

$w N$ - is the $n$ - th observation,

usually any scalar parameter $Q$ has to be calculated from these observations.

The question that arises is what precision is re- 
alizable from the observations $w$. In accordance with Cramer and Rao [3] $w$ is an amount of Fisher information about $\mathrm{Q}$, as following:

$$
\mathrm{m}=-\mathrm{E}\left[\frac{\partial^{2}\left(\ln \mathrm{f}_{\mathrm{N}}\right)}{\partial \Theta^{2}}\right]
$$

where:

$E$ - is the mathematical expectation (first moment),

$f N=f(w, \Theta)$ is the joint probability density function $(p d f)$ of the observations $w$.

This $p d f$ contains $\Theta$ as a parameter. The main inference that can be done is: the variance $\operatorname{var}(t)$ of any estimator $t$ of $\Theta$ cannot be smaller then the reciprocal of the amount of Fisher information, i.e.:

$$
\operatorname{var}(t) \text { i } m-1
$$

The term $m-1$ is called the Cramer-Rao Lower Bound (CRLB). These estimates are associated with the $p d f$ of the observations only, but not connected with a particular estimator.

The CRLB is therefore very useful as standard with which the precision of a particular estimation or measurement method can be compared.

It is very important in teaching to find ways of simplification. If a fundamental theory is simplified for didactic purposes, the statements should still be correct and not mislead the student to false ideas. It is particularly important at any teaching level (bachelor or master) to clearly indicate the assumptions and restrictions made, and the limits of validity of the simplifications. Teaching general principles is possible if such principles exist and are wellgrounded in certain logical frameworks.

It is useful to discuss the information and processing procedures of a $\mathrm{mP}$-based measurement system in terms of its architecture. A functional block diagram is shown in Fig. 3.

Another problems that are of high interest for the students are the robust approaches for measurement. In this respect, D - S ADC have recently become popular because they avoid many of the difficulties inherent to the conventional methods for $\mathrm{A} / \mathrm{D}$ and $\mathrm{D} / \mathrm{A}$ conversion.

Oversampling converters on one hand can use simple and relatively high-tolerance analog components, but on the other hand they require fast and complex digital signal processing stages. They modulate the analog input into a simple digital code, usually single-bit words, at a frequency much higher than the Nyquist rate.

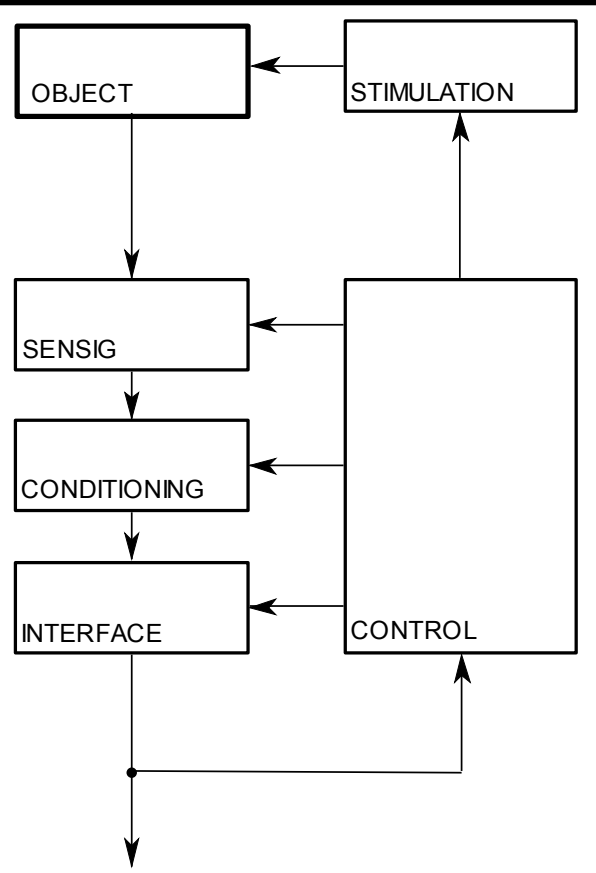

Fig. 3. Architecture of a mP-based measurement system

The aim, during lectures is to show how to reduce the noise in the signal band in High-Order Delta-Sigma Modulation.

This noise falls $3(2 L+1) d B$ for every doubling of the sampling rate, providing $(L+0,5)$ extra bits, where $L$ are the loops.

The lectures of DMS are designed for maximum flexibility in teaching. It is evident, the text that can be comprehended by the majority of students in a given specialization promotes student interest and involvement in the success of a subject in the auditorium. It is important to differentiate also between theories and laws. A law is an observed fact. A theory is an explanation of how or why a phenomenon occurs. The old idea that a law is a theory proved to be correct is false and has to be abandoned.

\section{THE LEARNING STRATEGY FOR THE STUDENTS' LABORATORY WORK}

The laboratory exercises are a learning-throughdoing program of activities. This statement is based on the philosophy that scientific knowledge is acquired through individual activity and experimentation. The tasks and the procedures are simple, so little interpretation is necessary. However, all activities are carried out under instructor supervision.

The main aim of such assignments (30h) is to model some measurement principles. Models are extremely valuable tools for scientists and engineers. They can be used to test hypotheses. They can predict effects and suggest precautions. In this respect, it is impor- 
tant to see the limitations of models. No model is exactly like that which it represents.

The students, during the same lab, are supposed to create high level and assembler language subroutines for control of various measurement procedures.

The hardware used in the already mentioned laboratory exercises are modules, produced by FEEDBACK company and IBM-compatible computers. This hardware and software created by the students makes possible modeling of different principles of Analog to Digital Conversion, measurement of various electrical and non-electrical quantities, calibration, investigation of the noise interference on the channels etc.

The efficient and effective study requires that students must be able to organize scientific data into a logical structure. In order to do this success fully, students must first be shown the organizational method used in the text and then be led to apply this method on their own.

A part of the labs is based on the software environment Classical Control(CC). Such an environment was developed at the California Institute of Technology for use in systems and control courses. The basic building blocks are Single-Input Single-Output transfer functions, either Laplace-transforms for analog systems or z-transforms for digital systems.

These blocks are used for investigation and for designing of analog channels of the Distributed Measurement Systems. The commands can be used to compute inverse transforms, convert between Laplace and z-domains, compute poles and zeros, and create any of the popular classical control graphs.

The environment $\mathrm{CC}$ can also be used for state space operations (with basic building blocks in a kind of state space quadruples). They can have multiple inputs and multiple outputs. The students can convert between analog and digital systems and to compute solutions to optimal control problems - the so-called optimization procedures.
The exercises of DMS are designed to focus on science content while reinforcing reading and math skills. Study skills and problem solving skills are the major areas of concentration.

\section{CONCLUSION}

This course is very useful for the students and increases their knowledge for practical applications of microproces sors and computers in measurement systems, distributed in a common environment.

\section{REFERENCES}

[1] I. Kalchev, I. Kodjabashev, N. Kolev, I. Petrov, T. Tashev, S. Yordanova - MEASUREMENT AND INSTRUMENTATION, Textbook for the students of English Language Department of Engineering, Published by TU-Sofia, 1998, (with ISBN 954-438-218-6), BULGARIA.

[2] D. Darjanova, N. Gurov, I. Kalchev, I. Kodjabashev, N. Kolev, V. Kolev, I. Petrov, P. Tsvetkov, T. Tashev - LABORATORY MANUAL ON MEASUREMENT AND INSTRUMENTATION, Textbook for the Students at the English Language Department of Engineering, Technical University of Sofia, 1999, Sofia, (with ISBN 954-438-251-8), BULGARIA.

[3] A. Stuart and J. Ord - Kendall's Advanced Theory Of Statistics, Vol. 2, Arnold, London, 1991.

[4] F. Mesch - "Didactic Reduction By Theory, With Special Attention To Measurement Education", J. Measurement, Vol. 14, No. 1(1994), pp. 15-22.

[5] O. Aumala, J. Holub and R. Smid - "Successful Virtual Conference", In: Proc. of the 6th ISMQC IMEKO Symposium - "METROLOGY FOR QUALITY CONTROL IN PRODUCTION”, Sept. 8-10, 1998, Wien AUSTRIA.

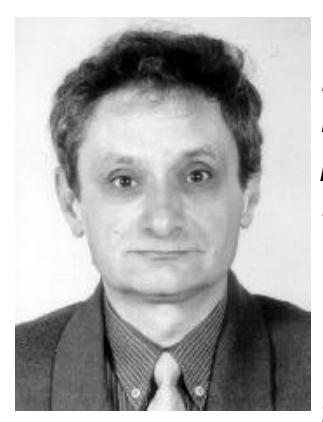

Ivan D. Kalchev (25 Oct. 1947), Bulgarian, graduated in computer science at the Kiev Politechnical Institute, Kiev, Ukraine (1971) and received the Ph.D. degree in measurement science from the Technical University of Sofia, Bulgaria (1991). He has published more than 100 research papers on microprocessor-based systems. Dr. Ivan Kalchev worked as associate researcher 10 years at the Central Institute for Computing Technique and Technologies - Sofia.

Became lecturer at the Department of Measurement, Technical University of Sofia (1993).

He teaches an university course of Electrical Measurements, and another of Distributed Measurement Systems. Since May, 1997 he is Associate Professor in measurement science at the TU - Sofia (Bulgaria). In 2002 Mr. I. Kalchev received the D.Sc. degree in technical sciences from the TU-Sofia. Award: "Prof. ITALO GORINI MEMORIAL AWARD" - Torino, ITALY, 1994. Areas of special interest: measurement and distributed instruments; statistics and probabilities theory; digital signal processing. 